\title{
Asthma and COVID-19: an update
}

\author{
Yochai Adir ${ }^{1,2}$, Walid Saliba ${ }^{2,3}$, Antoine Beurnier ${ }^{4,5,6}$ and Marc Humbert $\mathbb{1}^{4,5,6}$
}

${ }^{1}$ Pulmonary Division, Lady Davis Carmel Medical Center, Faculty of Medicine Technion Institute of Technology, Haifa, Israel. ${ }^{2}$ Ruth and Bruce Rappaport Faculty of Medicine, Technion-Israel Institute of Technology, Haifa, Israel. ${ }^{3}$ Dept of Community Medicine and Epidemiology, Lady Davis Carmel Medical Center, Haifa, Israel. ${ }^{4}$ Université Paris-Saclay, Le Kremlin-Bicêtre, France. ${ }^{5}$ Dept of Respiratory and Intensive Care Medicine, Assistance Publique-Hôpitaux de Paris (AP-HP), Hôpital Bicêtre, Le Kremlin-Bicêtre, France. ${ }^{6}$ INSERM, UMR_S 999, Hôpital Marie Lannelongue, Le Plessis-Robinson, France.

Corresponding author: Yochai Adir (adir-sh@zahav.net.il)

Shareable abstract (@ERSpublications)

Chronic or recurrent use of systemic corticosteroids before SARS-CoV-2 infection is a major risk factor of worst COVID-19 severity and survival in asthmatics as opposed to ICS and biological therapy which seems to be safe. https://bit.ly/3jU0zLR

Cite this article as: Adir Y, Saliba W, Beurnier A, et al. Asthma and COVID-19: an update. Eur Respir Rev 2021; 30: 210152 [DOI: 10.1183/16000617.0152-2021].

Copyright (CThe authors 2021

This version is distributed under the terms of the Creative Commons Attribution Non-Commercial Licence 4.0. For commercial reproduction rights and permissions contact permissions@ersnet.org

Received: 30 June 2021 Accepted: 3 Sept 2021

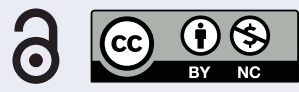

\section{Abstract}

As the world faces the coronavirus disease 2019 (COVID-19) pandemic due to severe acute respiratory syndrome coronavirus 2 (SARS-CoV-2) infection, concerns have been raised that asthma patients could be at increased risk of SARS-CoV-2 infection and disease severity. However, it appears that asthma is not an independent risk factor for both. Furthermore, asthma is not over-represented in hospitalised patients with severe pneumonia due to SARS-CoV-2 infection and there was no increased risk of asthma exacerbations triggered by SARS-CoV-2. There is accumulating evidence that asthma phenotypes and comorbidities are important factors in evaluating the risk for SARS-CoV-2 infection and disease severity, as findings suggest that Th2-high inflammation may reduce the risk of SARS-Cov-2 infection and disease severity in contrast to increased risk in patients with Th2-low asthma. The use of inhaled corticosteroids (ICS) is safe in asthma patients with SARS-CoV-2 infection. Furthermore, it has been proposed that ICS may confer some degree of protection against SARS-CoV-2 infection and the development of severe disease by reducing the expression of angiotensin converting enzyme-2 and transmembrane protease serine in the lung. In contrast, chronic or recurrent use of systemic corticosteroids before SARS-CoV-2 infection is a major risk factor of poor outcomes and worst survival in asthma patients. Conversely, biological therapy for severe allergic and eosinophilic asthma does not increase the risk of being infected with SARS-CoV-2 or having worse COVID-19 severity. In the present review we will summarise the current literature regarding asthma and COVID-19.

\section{Introduction}

Respiratory viruses are major drivers of acute exacerbations of asthma [1, 2]. As the world faces the coronavirus disease 2019 (COVID-19) pandemic due to the severe acute respiratory syndrome coronavirus 2 (SARS-CoV-2) infection, concerns have been raised regarding a possible increased risk of asthma exacerbations and COVID-19 severity in people with asthma. During the months following the beginning of the pandemic, studies were conducted in order to understand the relationship between asthma and the risk of SARS-CoV-2 infection, disease severity and prognosis. In the current review we will summarise the current literature regarding asthma and COVID-19.

Does asthma increase the risk for SARS-CoV-2 infection?

Angiotensin-converting enzyme-2 (ACE2) is the major receptor mediating the entry of SARS-CoV-2 into cells via its structural spike glycoprotein. The spike protein of SARS-CoV-2 is primed by transmembrane protease serine 2 (TMPRSS2), which allows fusion of viral and cellular membranes into host cells [3-6]. ACE2 expression is highly enriched in nasal epithelial cells and type II alveolar epithelial cells, and the expression of ACE2 in airways is regulated by airway inflammation and environmental stimuli, such as 
allergens and viruses [6-8]. An increased ACE2 expression exposes the lung to increased SARS-CoV-2 infectivity and COVID severity [9].

Epidemiological studies on the influenza A (H1N1) pandemics demonstrated that H1N1 infection was closely associated with asthma in both children and adults [10-12]. Furthermore, patients with asthma were more susceptible to asthma exacerbation and among patients hospitalised with pH1N1 virus infection in 2009, asthma was the most common underlying medical condition. Most of the asthmatic patients had an uncomplicated course of illness; however, severe disease, including intensive care unit admission and death, occurred in asthma patients who presented with pneumonia [10-12]. This experience led to a major concern that asthma would be one of most common risk factors for SARS-CoV-2 infection and COVID-19 severity and poor outcome. However, initial epidemiological studies reported that the incidence of COVID-19 in people with asthma was relatively low and that there was no apparent increased risk of SARS-CoV-2 infection those patients. Nevertheless, the reported prevalence of COVID-19 in people with asthma was diverse in different parts of the world (table 1) [13-27, 38, 46]. While early studies from China reported a relatively low prevalence of asthma among COVID-19 patients [13-17], some US data suggested a higher prevalence of COVID-19 in people with asthma when compared to the adult population (14.4\% versus $7.8 \%$ respectively) [18]. In an analysis of 22254 patients tested for SARS-CoV-2 (13442 of them with a positive PCR test for SARS-CoV-2), the prevalence of asthma patients among positive patients was $7 \%$, as compared to $11 \%$ in those with a negative PCR result [19]. Another analysis of 5700 patients hospitalised with COVID-19 in the New York City area, the prevalence of asthma and COPD were $9 \%$ and 5\%, respectively [20]. In Europe, the prevalence of asthma varied from country to country. In the UK, several studies reported higher asthma prevalence among patients with COVID-19 compared to the general population. In a large observational, population-based prospective cohort study using the International Severe Acute Respiratory and emerging Infection Consortium World Health Organization clinical characteristic protocol, the prevalence of asthma among 20133 hospitalised patients with COVID-19 was $14.5 \%$ [21]. Two studies that analysed data from the UK Biobank reported that the prevalence of asthma among patients with COVID-19 was $17.9 \%$ and $13 \%$, respectively [22, 23]. In contrast, in Italy and Sweden, epidemiological studies reported low asthma prevalence among patients with COVID-19 compared to the prevalence in the general population [24, 25]. In Latin America, an analysis of 89756 laboratory-confirmed COVID-19 cases from Mexico [26] reported low prevalence of asthma of 3.6\%; the reported prevalence in Brazil was even lower (1.6\%) [27]. Two recent real-life studies Korea and Spain reported a higher frequency of asthma in the COVID-19 population compared to the general COVID-19 population (2.9\% versus $1.6-2.2 \%$ and $1.41 \%$ versus $0.86 \%$, respectively) [28, 29]. Recently, a large cohort study from Israel reported that biologics approved for asthma and systemic corticosteroids (SCS) were not associated with increased risk of SARS-CoV-2 infection in asthma patients [30].

Several reasons may explain these differences in epidemiology. The method of asthma diagnosis is different between the studies, which may result in over or under diagnosis. Further, not all studies only included PCR-confirmed COVID-19 cases. Another possible explanation is different levels vulnerability to SARS-CoV-2 infection in different races or ethnic groups. PETERs et al. [31] found a higher expression of ACE2 in African Americans, which may be related to increased rate of SARS-CoV-2 infection. Furthermore, the prevalence of asthma in different counties is different, which may be related to

TABLE 1 Estimated asthma prevalence (\%) among patients with coronavirus disease 2019 (COVID-19) in comparison with the general population asthma prevalence in various countries $[13-27,38,46]$

\begin{tabular}{lcc} 
Country & National asthma prevalence (\%) & $\begin{array}{c}\text { Asthma prevalence among SARS-CoV-2 } \\
\text { positive patients (\%) }\end{array}$ \\
\hline China & $\sim 1.5-6.5$ & $\sim 1-1.5$ \\
USA & $\sim 8$ & $\sim 10-18$ \\
UK & $\sim 12-18$ & $\sim 14-18$ \\
Australia & $\sim 20$ & $\sim 10-14$ \\
Spain & $\sim 6-7$ & $\sim 5$ \\
Italy & $\sim 6$ & $\sim 1$ \\
Ireland & $\sim 9$ & $\sim 11$ \\
Switzerland & $\sim 5$ & $\sim 6.5$ \\
Germany & $\sim 7-8$ & $\sim 10-11$ \\
Israel & $\sim 7.5-8.5$ & $\sim 5$ \\
Mexico & $\sim 2.5$ & $\sim 2-3$ \\
Brazil & $\sim 12.5-13.0$ & $\sim 5-6$ \\
\hline
\end{tabular}


differences in environmental exposure, socioeconomic level and genetic predisposition. Furthermore, the prevalence among different ethnic groups is known to be different. In the US, for instance, the prevalence of asthma in African Americans is $10.6 \%$ while it is $7.6 \%$ in white Americans. Moreover, some authors hypothesised that the use of ICS could have a protective and therapeutic effect on COVID-19 [32]. ICS were found in in vitro studies to inhibit SARS-CoV-replication 2 in infected epithelial cells and reduced the expression of ACE2 and TMPRS in sputum epithelia cells [31-33]. Lastly, differences in local shielding recommendations, the obedience of the asthmatic population, different infection rates and the local policy of PCR testing may all affect the reported asthma prevalence among patients infected with SARS-CoV-2.

Overall, the current evidence supports the notion that asthma does not increase the risk of SARS-CoV-2 infection.

Does asthma increase the risk of severe COVID-19?

Older age and comorbidities, such as obesity, cardiovascular disease and diabetes, are considered as independent risk factors for severe COVID-19 and increased mortality [34]. Whether asthma should also be considered as an independent risk factor is less clear. The different studies included non-uniform asthmatic populations; therefore, diverse asthma severity, different asthma phenotypes and different background therapies such as ICS and SCS may explain the different levels of COVID-19 susceptibility and severity in asthma patients.

BEURNIER et al. [35] reported that, among hospitalised patients with severe pneumonia due to SARS-CoV-2 infection, asthma patients were not over-represented and patients with major comorbidities had worst outcomes. AvDEev et al. [36] described a low prevalence of asthma (1.8\%) among 1,307 intensive care unit patients with SARS-CoV-2 pneumonia who required mechanical ventilation. Based on prevalence data from 150 studies conducted worldwide, TeRRY et al. [37] found that there was no clear evidence of increased risk of COVID-19 diagnosis, hospitalisation, severity or mortality due to asthma. In a systemic review and metanalysis of 131 studies (410382 patients) Liu et al. [38] suggested that asthma was not associated with more severe COVID-19 phenotypes and poor outcomes. In addition, patients with asthma showed a tendency of lower death risk compared with patients without asthma, and no increase in the need for intubation/mechanical ventilation. In contrast, SchULTze et al. [39], using the OpenSAFELY platform, reported an increased risk of death from COVID-19 among asthma patients who had been prescribed high-dose ICS; however, the authors concluded that the observed increased risks of COVID-19-related death could be plausibly explained by unmeasured confounding factors such as disease severity and history of exacerbations, among other clinical factors which were not taken into account and might have influenced the results of the study. BLoom et al. [40] found that patients with asthma were more likely to receive critical care than patients without an underlying respiratory condition. In patients aged 16 years and older, severe asthma was associated with increased mortality compared to non-severe asthma.

A study in Korea included 7590 COVID-19 patients and reported that the mortality rate for COVID-19 patients with underlying asthma (7.8\%) was significantly higher than that of other patients (2.8\%; $\mathrm{p}<0.001$ ) [28]. However, after adjustment to other comorbidities, asthma was not found to be an independent risk factor for the clinical outcomes of COVID-19 [28]. A study from Spain also found an increased risk of hospitalisation due to COVID-19 in patients with asthma but it was largely associated with age and related comorbidities and mortality mainly affected elderly patients [29].

\section{Severe asthma and COVID-19}

The data on severe asthma and COVID-19 are scarce. A recent study from the Belgian Severe Asthma Registry reported a relatively low incidence of COVID-19 in patients with severe asthma and no association with a higher risk of SARS-CoV-2 infection or poor outcome [41]. HeffLER et al. [42] described the data from 1504 patients included in the Severe Asthma Network in Italy (SANI) and conclude that in patients with severe asthma COVID-19 was infrequent and was not an independent factor for severe COVID 19.

Consequently, based on the results of these and other studies, we may conclude that asthma including severe asthma is not and independent factor for increased COVID-19 severity and mortality.

Asthma phenotype and COVID-19

The epidemiological findings that asthma is not associated with increased risk for SARS-CoV-2 infection or severe COVID-19 raised the question whether a different asthma phenotype, mainly Th2-high and Th2-low, might have a different susceptibility to SARS-CoV-2 infection and severity. CAmIoLo et al. [43] 
reported that in a subset of patients with asthma which exhibits a high Th1 and a low Th2 epithelial gene expression signature in the bronchial epithelium, there is an increased expression of ACE2 receptor overlapped with type 1 and 2 interferon signatures, normally induced by viral infections [43]. These patients had other known risk factors for severe COVID-19, including male sex, history of hypertension and lymphopenia. The authors suggest that people with asthma with Th2-low inflammation may be at increased risk for adverse outcomes from COVID-19 [43]. In bronchial brush airway epithelial cells from asthmatic patients, BRADDING et al. [44] identified a positive correlation between ACE2 gene expression and a previously described interleukin (IL)-17-dependent gene expression signature, with an inverse association with Th2-high gene expression. In a Korean nationwide cohort, YANG et al. [45] reported that patients with non-allergic asthma had a greater risk of SARS-CoV-2 test positivity and severe clinical outcomes of COVID-19 than those with allergic asthma, supporting the idea that the Th2-low phenotype may be associated with an increased risk of severe COVID-19.

Many patients with Th2-low asthma are usually older with comorbidities such as obesity and diabetes and have a chronic subclinical inflammation due metabolic dysregulation. These priming factors of the immune system might expose those infected by SARS-CoV-2 to severe COVID-19 with a cytokine storm [46].

FERNANDO et al. [47] have suggested that a higher risk of hospitalisation in asthmatic women compared to asthmatic men could be due to a Th2-low immunological skewness, a predisposition towards more severe asthma, structural lung parenchymal differences and hormonal differences, which might increase asthmatic women's susceptibility to severe COVID-19, and in turn hospitalisation. However, ADIR et al. [48] analysed a large database of 214013 people with asthma indicating that the higher number of hospitalisations seen among asthmatic women was in fact due to a higher prevalence of comorbidities known to be independent risk factors for COVID-19 severity. Furthermore, subgroup analyses suggest that asthmatic women might have a lower risk of hospitalisation due to COVID-19 in the setting of hypertension, diabetes and obesity, when compared to asthmatic men [48].

Regarding Th2 inflammation, the current data are controversial. The immune responses to viruses are characterised by initial activation of innate immunity and production of interferons (IFN)- $\alpha / \beta$ and $-\lambda[49,50]$. Interestingly, a defective production of IFN by plasmacytoid dendritic and epithelial cells has been described in severe atopic patients with a consequent delayed and inefficient antiviral defence [51]. An important link was found between immunoglobulin E (IgE) levels and suppression of Toll-like receptor (TLR) 9-induced plasmacytoid dendritic cell IFN- $\alpha$ responses suggesting that in plasmacytoid dendritic cells, antiviral responses might be suppressed similarly in the setting of atopy [51, 52]. Furthermore, surface expression of the high affinity IgE receptor (FceRI) on plasmacytoid dendritic cells significantly correlates with serum IgE concentrations and is associated with diminished virus-induced IFN- $\alpha$ [53-55]. Interestingly. treatment with anti-IgE monoclonal antibody (omalizumab) may reduce plasmacytoid dendritic cell FceRI expression, suggesting improved antiviral responses [55].

JACKSON et al. [56] reported that ACE2 expression was significantly reduced in nasal and bronchial epithelial cells of patients with allergy and asthma. Furthermore, it was associated with allergen exposure, allergen sensitisation and high IgE levels. Conversely, nonatopic asthma was not associated with reduced ACE2 expression. Other studies reported that IL-4 and IL-13, which are characteristics of Th2-high/ eosinophilic asthma, can downregulate ACE2 expression in airway epithelial cells whereas tumour necrosis factor (TNF), IL-12 and IL-17A, which are cytokines highly produced in Th17/neutrophilic asthma, can upregulate ACE2 expression in vitro. Moreover, circulating soluble ACE2, which is upregulated in asthma, could act as a competitive interceptor, limiting the ability of SARS-CoV-2 to bind to airway cell membranes. In patients with Th2-high inflammation, ACE2 gene expression is downregulated [4, 57, 58]. Analysis of nasal airway transcriptome data from 695 children demonstrated that TMPRSS2 is highly upregulated by Th2 inflammation through the action of IL-13 [59]. However, there are very few cells co-expressing ACE2 and TMPRSS2 simultaneously. Therefore, it is questionable whether this increased TMPRSS2 expression favours SARS-CoV-2 infection in patients with allergic asthma [59]. Interestingly, WARK et al. [60] demonstrated reduced ACE2 expression in in lower bronchial epithelial cells in patients with asthma but did not find a difference in ACE2 expression with atopy or bronchial lavage eosinophil count. Furthermore, a reduced ACE2 expression was found to be associated with reduced furin expression in subjects with asthma in concert with increased expression of ADAM-17, which may suggest that IL-13 may downregulate ACE2 expression via ADAM-17 prior to infection with SARS-CoV-2.

Another unresolved question is whether Th2 inflammation affects the risk of severe COVID-19. Th2 inflammation downregulates the production of pro-inflammatory cytokines such as IL-1 $\beta$, TNF- $\alpha$ and IL-6, which are important in the defence against viral infections but also have a role in the late-phase 
hyper-inflammation that is a typical phase in COVID-19 leading to severe disease [51]. A recent study by ZHu et al. [23] based on data from the UK Biobank reported that adults with nonallergic asthma had a higher risk of severe COVID-19, whereas the risk of severe COVID-19 was not significantly elevated in patients with allergic asthma. Based on a nationwide database from Korea, YANG et al. [45] also reported that patients with nonallergic asthma had a greater risk of SARS-CoV-2 test positivity and severe clinical outcomes of COVID-19 than those with allergic asthma.

Interestingly, hospitalised patients with COVID-19 usually did not have asthma exacerbations, contrasting with findings in patients infected with other respiratory viruses which are important causes of asthma exacerbations [35]. One of the possible explanations for this is a high prevalence of Th2 inflammation, mainly in children, which has a protective effect on SARS-CoV-2 infectivity and disease severity.

Asthma medications and the risk for SARS-CoV-2 infection and disease severity The COVID-19 pandemic raises important questions regarding the use of ICS, SCS and biologics in asthma patients and the risk of increased SARS-CoV-2 infectivity or disease severity (figure 1).

\section{ICS}

ICS are used as anti-inflammatory controller therapy given either alone or in a combination with long-acting bronchodilators. The potential immunosuppressive effects of ICS, which may promote viral replication, delayed viral clearance and increased risks of secondary infections, raised concern regarding their use in asthma patients with COVID-19 [61, 62]. Furthermore, in vitro studies reported that ICS led to decreased rhinovirus elimination and decreased immune response to rhinoviral infection [61, 62]. In contrast, there are evidence to suggest that ICS may be beneficial in viral infections, specifically those due to coronavirus [63]. Previous studies have demonstrated that ICS reduced inflammatory cytokines levels such as IL-6 and IL-8 [64, 65]. In a retrospective analysis of data on 1520 patients hospitalised due to A/ H1N1, Myles et al. [66] found that asthma patients were less likely than non-asthma patients to have severe outcomes, which might be explained by pre-admission ICS use in asthma patients. In addition, pre-treatment of human respiratory epithelial cells in vitro with budesonide, in combination with glycopyrronium and formoterol, has inhibitory actions on coronavirus HCoV-229E replication and cytokine production [64]. Matsuyama et al. [33] reported that ciclesonide suppressed SARS-CoV-2 replication in cultured cells and inhibits SARS-CoV-2 cytopathic activity. In an analysis of gene expression for ACE2 and TMPRSS2 in sputum cells from 330 well-defined asthma patients, PETERs et al. [31] found a significantly lower expression of ACE2 and TMPRSS2 in patients treated with ICS. Most of the epidemiological studies have reported that there is no evidence that the use of ICS either increases SARS-CoV-2 infectivity and COVID-19 severity or has a beneficial effect on outcome. Using big-data analytics and artificial intelligence through the SAVANA Manager clinical platform, IzQuIERDo et al. [29] analysed clinical data from 71182 patients with asthma. They concluded that ICS showed a safe profile. Compared to asthma patients who required hospitalisation due to COVID-19, a significantly higher percentage of non-hospitalised patients used ICS [26]. CHог et al. [28] reported that ICS treatment was not found to be an independent factor for poor clinical outcomes of COVID-19. In contrast, using the

\begin{tabular}{|c|c|c|c|}
\hline & \\
\hline & Novious treatment & \\
\hline Infection with SARS-CoV-2 & No increased risk & No increased risk & No increased risk \\
\hline COVID-19 severity & Increased risk & No increased risk & No increased risk \\
\hline COVID-19 mortality & Increased risk & No increased risk & No increased risk \\
\hline
\end{tabular}

FIGURE 1 Asthma medications and the risk for severe acute respiratory syndrome coronavirus 2 (SARS-CoV-2) infection and disease severity. COVID-19: coronavirus disease 2019; ICS: inhaled corticosteroids; SCS: systemic corticosteroids. 
OpenSAFELY platform, SCHULTZE et al. [39] reported an increased risk of death from COVID-19 among people with asthma prescribed high-dose ICS. However, the authors suggest that this association could readily be explained by unmeasured confounders [39]. Interestingly, a large multi-centre prospective cohort study by BLOom et al. [40] reported that the use of ICS in patients aged 50 years and older within 2 weeks of admission was associated with decreased mortality. One major difference between the studies which may explain the discrepancy in the results is that in some of them the ICS treatment was taken as a whole and was not stratified to assess the effect of different doses of ICS.

As discussed, ICS have anti-inflammatory effects in the lungs, reduce expression of ACE-2 and TMPRSS2 in bronchial epithelial cells, and may reduce replication of SARS-CoV-2 in epithelial cells in vitro. Therefore, it was thus hypothesised that ICS might have a protective role in SARS-CoV-2 infection. In the Steroids in COVID-19 (STOIC) trial, an open-label, parallel-group, phase 2, randomised controlled trial, inhaled budesonide, when given to adults with early COVID-19, reduced the likelihood of requiring urgent care, emergency department consultation or hospitalisation, with faster symptom resolution [67]. This led to the PRINCIPLE trial in order to try to establish whether inhaled budesonide reduces time to recovery and COVID-19-related hospital admissions or deaths among people at high risk of complications in the community [68]. Inhaled budesonide reduced symptom burden and time to recovery, with a high probability of also reducing the need for hospital admission, albeit just below the prespecified superiority threshold in the primary analysis population.

Altogether, it seems that ICS are not an independent risk factor for increased SARS-CoV-2 infectivity or COVID-19 severity. ICS may have a role in the treatment of early COVID-19 in the community; however, larger studies are needed to better understand the effect of ICS on COVID-19 pathophysiology, the effectiveness of other ICS and the effect on fully vaccinated people with COVID-19.

SCS (figure 2)

The RECOVERY trial clearly showed that oral or intravenous administration of dexamethasone (at a dose of $6 \mathrm{mg}$ once daily; equal to prednisone $40 \mathrm{mg}$ ), for up to 10 days (or until hospital discharge if sooner), significantly reduces 28-day mortality among patients admitted to hospital with COVID-19 receiving invasive mechanical ventilation or oxygen [69]. The data on COVID-19 in patients receiving chronic or regular SCS therapy for asthma remain scarce. Using the OpenSAFELY platform to examine factors associated with COVID-19-related death, Williamson et al. [34] reported that severe asthma defined by

a)

No OCS (reference)

OCS use b)

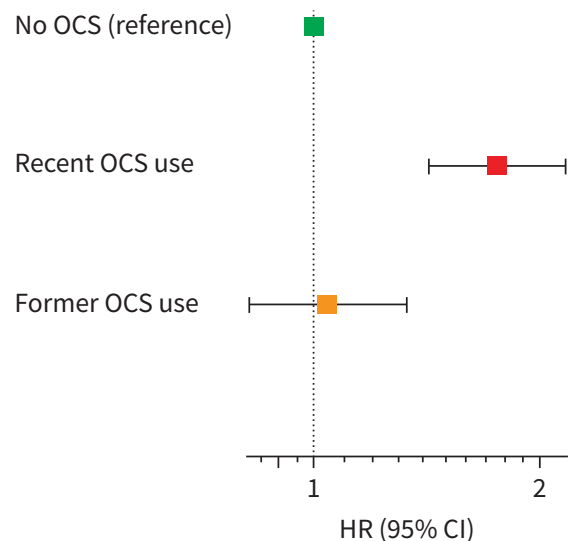

c)

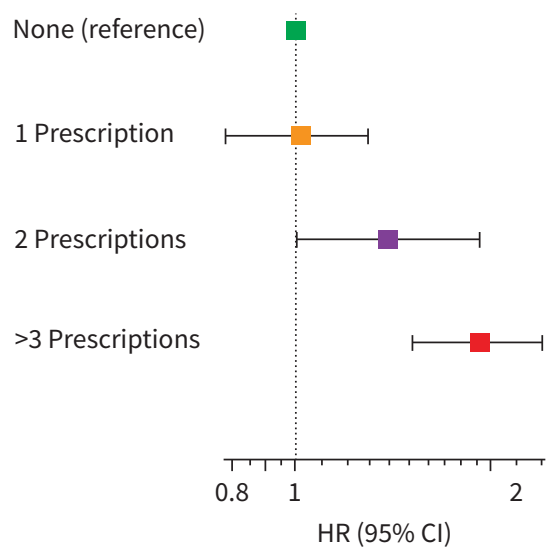

FIGURE 2 Association of oral corticosteroids use and outcomes in asthmatic patients with coronavirus disease 2019 (COVID-19). Reproduced from AdIR et al. [30]. a) Adjusted ${ }^{\#}$ hazard ratios (HRs; 95\% Cl) for the association oral corticosteroids (OCS) use in the prior year and the composite of moderate to severe COVID-19 or all-cause mortality within 90 days following PCR date among adult asthmatic patients with positive PCR for severe acute respiratory syndrome coronavirus 2 (SARS-CoV-2) ( $n=8242$ ). OCS use refers to any use in the year before baseline with no OCS use serving as reference category. b) Adjusted ${ }^{\#}$ HRs $(95 \% \mathrm{Cl})$ for the association recent and former OCS use and the composite of moderate to severe COVID-19 or all-cause mortality within 90 days following PCR date among adult asthmatic patients with positive PCR for SARS-CoV-2 ( $n=8242$ ). c) Adjusted ${ }^{\#}$ HRs $(95 \% \mathrm{Cl})$ for the association between the number of filled steroids prescriptions in the prior years and the composite of moderate to severe COVID-19 disease and all-cause mortality within 90 days following PCR date among adult asthmatic patients with positive PCR for SARS-CoV-2 $(\mathrm{n}=8242) . .^{\#}$ : adjusted for age, sex, ethnicity, diabetes, hypertension, ischaemic heart disease, obesity, smoking and biologics use. 
recent SCS use (less than 1 year from the day of SARS-CoV-2 infection, no details of the form or dose of the SCS were given), was associated with increased mortality. In a smaller group of 15 asthma patients who received SCS (13 of them in the 2 weeks before COVID-19 diagnosis), CHнIBA et al. [70] reported that SCS (short course of prednisone, doses are not mentioned), was not associated with COVID-19-related hospitalisation. A recent large nationwide study of 80602 adult asthma patients in Israel, reported that chronic SCS use (oral prednisone 5-40 mg per day) as well as recent use (defined as within less than 120 days) and more than two prescriptions of SCS (up to $40 \mathrm{mg}$ a day) for 3 days or more for asthma exacerbation during the last year were all an independent risk factor for worst COVID-19 severity and all-cause mortality [30]. Conversely, SCS use did not increase the likelihood of being infected with SARS-CoV-2 [30]. There is no clear explanation why treatment with SCS is found to be an independent risk factor for disease severity and mortality. The evidence supporting the use of SCS in other causes of viral pneumonia are weak, mainly due to differences in the dose of SCS used, disease severity and medical condition [71-73]. In the RECOVERY trial [69], the positive effect of SCS on mortality may be explained by the later stage of SCS administration where the inflammation process is dominant and active viral replication is low. On the other hand, the need for SCS to treat and/or prevent exacerbations and to improve asthma control define a group of patients with severe uncontrolled asthma which are at increased risk for severe COVID-19 and poor outcome. In conclusion, the use of SCS, whether for chronic, recent or for recurrent exacerbations, is a clear risk factor for increased COVID-19 severity and mortality.

\section{Biologics}

Biological therapy approved for asthma include anti-IgE, anti-IL-5/anti-IL-5R and anti-IL4R $\alpha$ which are indicated for patients with uncontrolled severe allergic/eosinophilic asthma, with recurrent exacerbations [74]. These biologics block pathways of Th2 inflammation, which might have a protective effect against COVID-19 as indicated above. Therefore, concerns have been raised whether the use of biologics may be associated of increased risk of SARS-CoV-2 infection or increased COVID-19 severity [51, 56].

Eosinophils have a role in fighting viral infections, especially against RNA viruses [75-77]. TLR7, which recognises viruses and single-stranded RNA, is one of the most important viral receptors in eosinophils, underscoring the role of eosinophils in virus recognition [78]. Eosinopenia was found in patients presenting with moderate-to-severe COVID-19 and is typically accompanied by reductions in peripheral lymphocyte, platelet and monocyte counts [79-82]. Furthermore, eosinophil counts have been included in several algorithms used to predict disease severity [83, 84]. FerastraOaru et al. [85] found that pre-existing eosinophilia ( $\geqslant 150$ cells $\cdot \mu \mathrm{L}^{-1}$ ) in asthmatic patients was protective from COVID-19-associated admission, and development of eosinophilia $\left(\geqslant 150\right.$ cells $\left.\mu \mathrm{L}^{-1}\right)$ during hospitalisation was associated with decreased mortality. Ho et al. [86] reported that blood eosinophils greater than or equal to $200 \mathrm{cells} \cdot \mathrm{mL}^{-1}$, both in those with and without asthma, may be associated with reduced mortality risk. Eosinophils are the major target of the three anti-IL5/IL5R asthma biologics, mepolizumab, reslizumab and benralizumab, which cause either reduction or depletion of tissue and peripheral blood eosinophils. Therefore, the major concern is whether reducing the number of eosinophils and their products may affect the susceptibility or severity of SARS-CoV-2 infection.

However, the clinical evidence shows that the use of biologics is safe [73]. In a study by ADIR et al. [30], a significantly lower eosinophil count $\left(42 \pm 39 \cdot \mu \mathrm{L}^{-1}\right)$ in patients treated by biologics was not found to be associated with increased COVID-19 severity and mortality.

IZQUIERDO et al. [29] reported that the incidence of COVID-19 was higher among those on biologics compared to the general asthma population (2.3\% versus $1.4 \%)$, but that the number of COVID-19-related admissions and mortality in these patients was low. Use of biologic therapy was not associated with COVID-19 severity in the Severe Asthma Network in Italy and the Belgian Severe Asthma Registry [27, 28]. In a cross-sectional telephone-based survey including 473 patients on biological therapy, MatUCCI et al. [87] found no increased risk for COVID-19. A Spanish study of 545 patients with severe asthma on biologics treatment reported no increased risk of COVID-19 infection or greater disease severity and mortality [88]. Recently, ADIR et al. [30] did not find an increased risk for SARS-CoV-2 infection or increased COVID-19 severity in a large cohort of 514 asthma patients on biologics.

Previous studies have reported a close association between allergic sensitisation and the risk of acute virus-induced wheezing episodes [89-91]. Furthermore, allergic inflammation can impair antiviral responses and in early childhood allergic sensitisation is followed by viral wheezing [53, 92]. These findings suggest that mechanisms involving IgE might reduce antiviral responses. Indeed, several studies reported that treatment with anti-IgE-omalizumab was able to reduce the duration of rhinovirus infections, viral shedding and risk of rhinovirus illnesses [74, 94, 95]. Heymann et al. [95] demonstrated that blocking 
IgE with omalizumab resulted in a marked reduction in the percentage of asthma patients who experienced lower respiratory tract symptoms. In the PROSE study the investigators found that inhibition of IgE reduced morbidity caused by naturally acquired rhinovirus infections and that these effects include reductions in viral illnesses, viral asthma exacerbations, and the duration and peak of viral shedding [95]. However, there is no clinical evidence that omalizumab reduced or increased the risk of SARS-CoV-2 infectivity or disease severity [30]. Dupilumab is a humanised mAb directed against the alpha chain of the IL4 receptor shared by both IL4 and IL13. The study by ADIR et al. [30] included 60 patients on dupilumab. Again, no increased risk for SARS-COV-2 infection or COVID-19 severity was found. Overall, the current evidence suggests that biological treatment for severe allergic and eosinophilic asthma does not increase the risk for SARS-CoV-2 infection or COVID-19 severity.

\section{Conclusion}

Although at the beginning of the COVID-19 pandemic there were concerns that asthma patients could be at increased risk for SARS-CoV-2 infection and disease severity, it appears that asthma is not an independent risk factor for both. Furthermore, asthma is not over-represented in hospitalised patients with severe pneumonia due to SARS-CoV-2 infection and there has been no increased risk of asthma exacerbations triggered by SARS-CoV-2 in that setting. Interestingly, a large difference in the incidence of COVID-19 in asthma patients was found in different geographical areas. This difference might be explained by several factors including a different vulnerability to COVID-19 infection and differences in asthma prevalence in different races or ethnic groups. There is accumulating evidence that asthma phenotypes are important factors in evaluating the risk for SARS-CoV-2 infection and disease severity, as findings suggest that Th2-high inflammation may reduce the risk of SARS-Cov-2 infection and disease severity in contrast to increased risk in patients with Th2-low asthma. Regarding asthma medications, the use of ICS, despite early concern about immunosuppression, is safe. Furthermore, ICS do not increase infectivity or disease severity. In contrast, chronic or recurrent use of SCS before SARS-CoV-2 infection is a major risk factor for poor outcomes and worst survival. Biological therapy for severe allergic and eosinophilic asthma does not increase the risk of being infected with SARS-CoV-2 or having worse COVID-19 severity. These data emphasise the need for optimised management of asthma patients in order to achieve asthma control and avoid whenever possible the need for chronic or recurrent use of SCS. However, further studies are needed to answer still unresolved questions such as the relation between different asthma phenotypes and SARS-CoV-2 infection.

Provenance: Commissioned article, peer reviewed.

Conflict of interest: Y. Adir reports personal fees from Teva, grants and personal fees from GSK, grants and personal fees from AstraZeneca, personal fees from Sanofi, outside the submitted work. W. Saliba has nothing to disclose. A. Beurnier reports personal fees from AstraZeneca, personal fees from Sanofi, outside the submitted work. M. Humbert reports personal fees from Acceleron, grants and personal fees from Actelion, grants and personal fees from Bayer, personal fees from GSK, personal fees from Merck, personal fees from Novartis, personal fees from AstraZeneca, personal fees from Sanofi, outside the submitted work.

\section{References}

1 Satia I, Cusack R, Greene JM, et al. Prevalence and contribution of respiratory viruses in the community to rates of emergency department visits and hospitalizations with respiratory tract infections, chronic obstructive pulmonary disease and asthma. PLOS ONE 2020; 15: e0228544.

2 Busse WW, Lemanske Jr RF, Gern JE. Role of viral respiratory infections in asthma and asthma exacerbations. Lancet 2010; 376: 826-834.

3 Walls AC, Park YJ, Tortorici MA, et al. Structure, function, and antigenicity of the SARS-CoV-2 spike glycoprotein. Cell 2020; 181: 281-292.

4 Branco ACCC, Sato MN, Alberca RW. The possible dual role of the ACE2 receptor in asthma and coronavirus (SARS-CoV2) infection. Front Cell Infect Microbiol 2020; 10: 550571.

5 Zhou P, Yang X-L, Wang X, et al. A pneumonia outbreak associated with a new coronavirus of probable bat origin. Nature 2020; 579: 270-273.

6 Hoffmann M, Kleine-Weber H, Schroeder S, et al. SARS-CoV-2 cell entry depends on ACE2 and TMPRSS2 and is blocked by a clinically proven protease inhibitor. Cell 2020; 181: 271-280.

7 Li G, He X, Zhang L, et al. Assessing ACE2 expression patterns in lung tissues in the pathogenesis of COVID-19. J Autoimmun 2020; 112: 102463.

8 Sungnak W, Huang N, Bécavin C, et al. SARS-CoV-2 entry factors are highly expressed in nasal epithelial cells together with innate immune genes. Nat Med 2020; 26: 681-687. 
9 Yao Y, Wang H, Liu Z. Expression of ACE2 in airways: implication for COVID-19 risk and disease management in patients with chronic inflammatory respiratory diseases. Clin Exp Allergy 2020; 50: 1313-1324.

10 McKenna JJ, Bramley AM, Skarbinski J, et al. Asthma in patients hospitalized with pandemic influenza A (H1N1)pdm09 virus infection-United States, 2009. BMC Infect Dis 2013; 13: 57.

11 Nguyen-Van-Tam JS, Openshaw PJ, Hashim A, et al. Risk factors for hospitalisation and poor outcome with pandemic A/H1N1 influenza: United Kingdom first wave (May-September 2009). Thorax 2010; 65: 645-651.

12 Obuchi M, Adachi Y, Takizawa T, et al. Influenza A(H1N1)pdm09 virus and asthma. Front Microbiol 2013; 4: 307.

13 Yang J, Zheng Y, Gou X, et al. Prevalence of comorbidities and its effects in patients infected with SARS-CoV-2: a systematic review and meta-analysis. Int J Infect Dis 2020; 94: 91-95.

14 Wu Z, McGoogan JM. Characteristics of and important lessons from the coronavirus disease 2019 (COVID-19) outbreak in China: summary of a report of 72314 cases from the Chinese Center for Disease Control and Prevention. JAMA 2020; 323: 1239-1242.

15 Li X, Xu S, Yu M, et al. Risk factors for severity and mortality in adult COVID-19 inpatients in Wuhan. $J$ Allergy Clin Immunol 2020; 146: 110-118.

16 Zhang J-J, Dong X, Cao Y-Y, et al. Clinical characteristics of 140 patients infected with SARS-CoV-2 in Wuhan, China. Allergy 2020; 75: 1730-1741.

17 Zhang J-J, Cao Y-Y, Dong X, et al. Distinct characteristics of COVID-19 patients with initial rRT-PCR-positive and rRT-PCR-negative results for SARS-CoV-2. Allergy 2020; 75: 1809-1812.

18 Centers for Disease Control and Prevention. Asthma prevalence. www.cdc.gov/asthma/data-visualizations/ prevalence.htm. Accessed: 13 July 2020.

19 Kalyanaraman Marcello R, Dolle J, Grami S, et al. Characteristics and outcomes of COVID-19 patients in New York City's public hospital system. PLoS ONE 2020; 15: e0243027.

20 Richardson S, Hirsch JS, Narasimhan M, et al. Presenting characteristics, comorbidities, and outcomes among 5700 patients hospitalized with COVID-19 in the New York City area. JAMA 2020; 323: 2052-2059.

21 Docherty AB, Harrison EM, Green CA, et al. Features of 16,749 hospitalised UK patients with COVID-19 using the ISARIC WHO Clinical Characterisation Protocol. BMJ 2020; 369: m1985.

22 Khawaja AP, Warwick AN, Hysi PG, et al. Associations with COVID-19 hospitalisation amongst 406,793 adults. The UK Biobank prospective cohort study. medRxiv 2020; preprint [https://doi.org/10.1101/2020.05.06. 20092957].

23 Zhu Z, Hasegawa K, Ma B, et al. Association of asthma and its genetic predisposition with the risk of severe COVID-19. J Allergy Clin Immunol 2020; 146: 327-329.e4.

24 Gemes K, Talbäck M, Modig K, et al. Burden and prevalence of prognostic factors for severe COVID-19 in Sweden. Eur J Epidemiol 2020; 35: 401-409.

25 Caminati M, Lombardi C, Micheletto C, et al. Asthmatic patients in COVID-19 outbreak: few cases despite many cases. J Allergy Clin Immunol 2020; 146: 541-542.

26 Solís P, Carreno H. COVID-19 fatality and comorbidity risk factors among confirmed patients in Mexico. medRxiv 2020; preprint [https://doi.org/10.1101/2020.04.21.20074591].

27 Rezende LFM, Thome B, Schveitzer MC, et al. Adults at high-risk of severe coronavirus disease-2019 (COVID-19) in Brazil. Revista Saude Publica 2020; 54: 50.

28 Choi YJ, Park J-Y, Lee HS, et al. Effect of asthma and asthma medication on the prognosis of patients with COVID-19. Eur Respir J 2021; 57: 2002226.

29 Izquierdo JL, Almonacid C, González Y, et al. The impact of COVID-19 on patients with asthma. Eur Respir J 2021; 57: 2003142

30 Adir Y, Humbert M, Saliba W. COVID-19 risk and outcomes in adult asthmatics treated with biologics or systemic corticosteroids: nationwide real-world evidence. J Allergy Clin Immunol 2021; 15: S0091-6749(21) 00938-6.

31 Peters MC, Sajuthi S, Deford P, et al. COVID-19 related genes in sputum cells in asthma: relationship to demographic features and corticosteroids. Am J Respir Crit Care Med 2020; 202: 83-90

32 Halpin DMG, Singh D, Hadfield RM. Inhaled corticosteroids and COVID-19: a systematic review and clinical perspective. Eur Respir J 2020; 55: 2001009.

33 Matsuyama S, Kawase M, Nao N, et al. The inhaled steroid ciclesonide blocks SARS-CoV-2 RNA replication by targeting the viral replication-transcription complex in cultured cells. J Virol 2020; 95: e01648-20.

34 Williamson EJ, Walker AJ, Bhaskaran K, et al. Factors associated with COVID-19-related death using OpenSAFELY. Nature 2020; 584: 430-436.

35 Beurnier A, Jutant EM, Jevnikar M, et al. Characteristics and outcomes of asthmatic patients with COVID-19 pneumonia who require hospitalisation. Eur Respir J 2020; 56: 2001875.

36 Avdeev S, Moiseev S, Brovko M, et al. Low prevalence of bronchial asthma and chronic obstructive lung disease among intensive care unit patients with COVID-19. Allergy 2020; 75: 2703-2704.

37 Terry PD, Heidel RE, Dhand R. Asthma in adult patients with COVID-19: prevalence and risk of severe disease. Am J Respir Crit Care Med 2021; 203: 893-905. 
38 Liu S, Cao Y, Du T, et al. Prevalence of comorbid asthma and related outcomes in COVID-19: a systematic review and meta-analysis. J Allergy Clin Immunol Pract 2021; 9: 693-701.

39 Schultze A, Walker AJ, Mackenna B, et al. Risk of COVID-19-related death among patients with chronic obstructive pulmonary disease or asthma prescribed inhaled corticosteroids: an observational cohort study using the OpenSAFELY platform. Lancet Respir Med 2020; 8: 1106-1120.

40 Bloom Cl, Drake TM, Docherty AB, et al. Risk of adverse outcomes in patients with underlying respiratory conditions admitted to hospital with COVID-19: a national, multicentre prospective cohort study using the ISARIC WHO Clinical Characterisation Protocol UK. Lancet Respir Med 2021; 9: 699-711

41 Hanon S, Brusselle G, Deschampheleire M, et al. COVID-19 and biologics in severe asthma: data from the Belgian Severe Asthma Registry. Eur Respir J 2020; 56: 2002857.

42 Heffler E, Detoraki A, Contoli M, et al. COVID-19 in Severe Asthma Network in Italy (SANI) patients: Clinical features, impact of comorbidities and treatments. Allergy 2021; 76: 887-892.

43 Camiolo M, Gauthier M, Kaminski N, et al. Expression of SARS-CoV-2 receptor ACE2 and coincident host response signature varies by asthma inflammatoryenotype. J Allergy Clin Immunol 2020; 146: 315-324.

44 Bradding P, Richardson M, Hinks TSC, et al. ACE2, TMPRSS2, and furin gene expression in the airways of people with asthma-implications for COVID-19. J Allergy Clin Immunol 2020; 146: 208-211.

45 Yang JM, Koh HY, Moon SY, et al. Allergic disorders and susceptibility to and severity of COVID-19: A nationwide cohort study. J Allergy Clin Immunol 2020; 146: 790-798.

46 Skevaki C, Karsonova A, Karaulov A, et al. Asthma-associated risk for COVID-19 development. J Allergy Clin Immunol 2020; 146: 1295-1301.

47 Fernando M, Agusti A, Dharmage S, et al. Are women with asthma at increased risk for severe COVID-19? Lancet Respir Med 2021; 9: 125-126.

48 Adir Y, Humbert M, Saliba W. Are women with asthma at increased risk for severe COVID-19? Lancet Respir Med 2021; 9: E75-E76.

49 Liu S, Zhi Y, Ying S. COVID-19 and asthma: reflection during the pandemic. Clin Rev Allergy Immunol 2020; 59: 78-88.

50 Zhu J, Message SD, Mallia P, et al. Bronchial mucosal IFN- $\alpha / \beta$ and pattern recognition receptor expression in patients with experimental rhinovirus-induced asthma exacerbations. J Allergy Clin Immunol 2019; 143: 114-125.

51 Carli G, Cecchi L, Stebbing J, et al. Is asthma protective against COVID-19? Allergy 2021; 76: 866-868.

52 Maggi E, Parronchi P, Manetti R, et al. Reciprocal regulatory effects of IFN-gamma and IL-4 on the in vitro development of human Th1 and Th2 clones. J Immunol 1992; 148: 2142-2147.

53 Gill MA, Bajwa G, George TA, et al. Counterregulation between the FceRI pathway and antiviral responses in human plasmacytoid dendritic cells. J Immunol 2010; 184: 5999-6006.

54 Durrani SR, Montville DJ, Pratt AS, et al. Innate immune responses to rhinovirus are reduced by the high-affinity IgE receptor in allergic asthmatic children. J Allergy Clin Immunol 2012; 130: 489-495

55 Gill MA, Liu AH, Calatroni A, et al. Enhanced plasmacytoid dendritic cell antiviral responses after omalizumab. J Allergy Clin Immunol 2018; 141: 1735-1743.

56 Jackson DJ, Busse WW, Bacharier LB, et al. Association of respiratory allergy, asthma, and expression of the SARS-CoV-2 receptor ACE2. J Allergy Clin Immunol 2020; 146: 203-206.

57 Ramakrishnan RK, Al Heialy S, Hamid Q. Implications of preexisting asthma on COVID-19 pathogenesis. Am J Physiol Lung Cell Mol Physiol 2021; 320: L880-L891.

58 Medina-Enríquez MM, Lopez-León S, Carlos-Escalante JA, et al. ACE2: the molecular doorway to SARS-CoV-2. Cell Biosci 2020; 10: 148.

59 Sajuthi SP, DeFord P, Jackson ND, et al. Type 2 and interferon inflammation strongly regulate SARS-CoV-2 related gene expression in the airway epithelium. Nat Commun 2020; 11: 5139.

60 Wark PAB, Pathinayake PS, Kaiko G, et al. ACE2 expression is elevated in airway epithelial cells from older and male healthy individuals but reduced in asthma. Respirology 2021; 26: 442-451.

61 Singanayagam A, Glanville N, Girkin JL, et al. Corticosteroid suppression of antiviral immunity increases bacterial loads and mucus production in COPD exacerbations. Nat Commun 2018; 9: 2229.

62 Yang $\mathrm{M}$, Zhang $\mathrm{Y}$, Chen $\mathrm{H}$, et al. Inhaled corticosteroids and risk of upper respiratory tract infection in patients with asthma: a meta-analysis. Infection 2019; 47: 377-385.

63 Lipworth B, Chan R, Kuo CR. Use of inhaled corticosteroids in asthma and coronavirus disease 2019: Keep calm and carry on. Ann Allergy Asthma Immunol 2020; 125: 503-504.

64 Yamaya M, Nishimura H, Deng X. Inhibitory effects of glycopyrronium, formoterol, and budesonide on coronavirus HCoV-229E replication and cytokine production by primary cultures of human nasal and tracheal epithelial cells. Respir Investig 2020; 58: 155-168.

65 Suda K, Tsuruta M, Eom J. Acute lung injury induces cardiovascular dysfunction: effects of IL-6 and budesonide/formoterol. Am J Respir Cell Mol Biol 2011; 45: 510-516.

66 Myles P, Nguyen-Van-Tam JS, Semple MG, et al. Influenza Clinical Information Network FLU-CIN. Differences between asthmatics and nonasthmatics hospitalised with influenza A infection. Eur Respir J 2013; 41: 824-831. 
67 Ramakrishnan S, Nicolau DV, Jr, Langford B, et al. Inhaled budesonide in the treatment of early COVID-19 (STOIC): a phase 2, open-label, randomised controlled trial. Lancet Respir Med 2021; 9: 763-772.

$68 \mathrm{Yu}$ LM, Bafadhel M, Dorward J, et al. Inhaled budesonide for COVID-19 in people at high risk of complications in the community in the UK (PRINCIPLE): a randomised, controlled, open-label, adaptive platform trial. Lancet 2021; 398: 843-855.

69 RECOVERY Collaborative Group, Horby P, Lim WS, et al. Dexamethasone in hospitalized patients with COVID-19. N Engl J Med. 2021; 384: 693-704.

70 Chhiba KD, Patel GB, Vu THT, et al. Prevalence and characterization of asthma in hospitalized and nonhospitalized patients with COVID-19. J Allergy Clin Immunol 2020; 146: 307-314.

71 Rabi YM, Mandourah Y, Al-Hameed F, et al. Corticosteroid therapy for critically ill patients with middle east respiratory syndrome. Am J Respir Crit Care Med 2018; 197: 757-767.

72 Lansbury LE, Rodrigo C, Leonardi-Bee J, et al. Corticosteroids as adjunctive therapy in the treatment of influenza: an updated Cochrane systematic review and meta-analysis. Crit Care Med 2020; 48: e98-e106.

73 Cheng PK, Wong DA, Tong LK, et al. Viral shedding patterns of coronavirus in patients with probable severe acute respiratory syndrome. Lancet 2004; 363: 1699-1700.

74 Morais-Almeida M, Aguiar R, Martin B, et al. COVID-19, asthma, and biological therapies: what we need to know. World Allergy Organ J 2020; 13: 100126.

75 Nagase H, Okugawa S, Ota Y, et al. Expression and function of Toll-like receptors in eosinophils: activation by Toll-like receptor 7 ligand. J Immunol 2003; 171: 3977-3982.

76 Wong CK, Cheung PF, Ip WK, et al. Intracellular signaling mechanisms regulating toll-like receptor-mediated activation of eosinophils. Am J Respir Cell Mol Biol 2007; 37: 85-96.

77 Drake MG, Bivins-Smith ER, Proskocil BJ, et al. Human and mouse eosinophils have antiviral activity against parainfluenza virus. Am J Respir Cell Mol Biol 2016; 55: 387-394.

78 Rosenberg HF, Foster PS. Eosinophils and COVID-19: diagnosis, prognosis, and vaccination strategies. Semin Immunopathol 2021; 43: 383-392.

79 Liao D, Zhou F, Luo L, et al. Haematological characteristics and risk factors in the classification and prognosis evaluation of COVID-19: a retrospective cohort study. Lancet Haematol 2020; 7: e671-e678.

80 Formica V, Minieri M, Bernardini S, et al. Complete blood count might help to identify subjects with high probability of testing positive to SARS-CoV-2. Clin Med 2020; 20: e114-e119.

81 Zhao L, Zhang YP, Yang X. Eosinopenia is associated with greater severity in patients with coronavirus disease 2019. Allergy 2021; 76: 562-564.

82 Danwang C, Endomba FT, Nkeck JR, et al. A meta-analysis of potential biomarkers associated with severity of coronavirus disease 2019 (COVID-19). Biomark Res 2020; 8: 37.

83 Ma J, Shi X, Xu W, et al. Development and validation of a risk stratification model for screening suspected cases of COVID-19 in China. Aging 2020; 12: 13882-13894.

84 Tordjman M, Mekki A, Mali RD, et al. Pre-test probability for SARS-CoV-2-related infection score: the PARIS score. PLOS ONE 2020; 15: e0243342.

85 Ferastraoaru D, Hudes G, Jerschow E, et al. Eosinophilia in asthma patients is protective against severe COVID-19 illness. J Allergy Clin Immunol Pract 2021; 9: 1152-1162.

86 Ho KS, Howell D, Rogers L, et al. The relationship between asthma, eosinophilia, and outcomes in coronavirus disease 2019 infection. Ann Allergy Asthma Immunol 2021; 127: 42-48.

87 Matucci A, Caminati M, Vivarelli E, et al. COVID-19 in severe asthmatic patients during ongoing treatment with biologicals targeting type 2 inflammation: Results from a multicenter Italian survey. Allergy 2021; 76: 871-874.

88 Rial MJ, Valverde M, Del Pozo V, et al. Clinical characteristics in 545 patients with severe asthma on biological treatment during the COVID-19 outbreak. J Allergy Clin Immunol Pract 2021; 9: 487-489.

89 Duff AL, Pomeranz ES, Gelber LE, et al. Risk factors for acute wheezing in infants and children: viruses, passive smoke, and IgE antibodies to inhalant allergens. Pediatrics 1993; 92: 535-540.

90 Kercsmar CM, Liu AH, Makhija MM, et al. Seasonal risk factors for asthma exacerbations among inner-city children. J Allergy Clin Immunol 2015; 135: 1465-1473.

91 Inoue Y, Shimojo N. Epidemiology of virus-induced wheezing/asthma in children. Front Microbiol 2013; 4: 391.

92 Soto-Quiros M, Avila L, Platts-Mills TA, et al. High titers of IgE antibody to dust mite allergen and risk for wheezing among asthmatic children infected with rhinovirus. J Allergy Clin Immunol 2012; 129: 1499-1505.

93 Esquivel A, Busse WW, Calatroni A, et al. Effects of omalizumab on rhinovirus infections, illnesses, and exacerbations of asthma. Am J Respir Crit Care Med 2017; 196: 985-992.

94 Altman MC, Beigelman A, Ciaccio C, et al. Evolving concepts in how viruses impact asthma: a Work Group Report of the Microbes in Allergy Committee of the American Academy of Allergy, Asthma \& Immunology. J Allergy Clin Immunol 2020; 145: 1332-1344.

95 Heymann PW, Platts-Mills TA, Woodfolk JA, et al. Understanding the asthmatic response to an experimental rhinovirus infection: exploring the effects of blocking IgE. J Allergy Clin Immunol 2020; 146: 545-554. 